\title{
BMJ Open Adverse childhood experiences (ACEs) and later-life depression: perceived social support as a potential protective factor
}

\author{
E Von Cheong, ${ }^{1}$ Carol Sinnott, ${ }^{2}$ Darren Dahly, ${ }^{3}$ Patricia M Kearney ${ }^{3}$
}

To cite: Cheong EV, Sinnott C, Dahly D, et al. Adverse childhood experiences (ACEs) and later-life depression: perceived social support as a potential protective factor. BMJ Open 2017;7:e013228. doi:10.1136/ bmjopen-2016-013228

- Prepublication history and additional material for this paper are available online. To view these files please visit the journal online (http://dx.doi. org/10.1136/bmjopen-2016013228).

Received 28 June 2016 Revised 26 March 2017 Accepted 29 March 2017

CrossMark

${ }^{1}$ School of Medicine, University College Cork, Cork, Ireland ${ }^{2}$ Department of General Practice, University College Cork, Cork, Ireland

${ }^{3}$ Department of Epidemiology and Public Health, University College Cork, Cork, Ireland

Correspondence to Dr E Von Cheong; 111123401@umail.ucc.ie

\section{ABSTRACT}

Objective To investigate associations between adverse childhood experiences (ACEs) and later-life depressive symptoms; and to explore whether perceived social support (PSS) moderates these.

Method We analysed baseline data from the Mitchelstown (Ireland) 2010-2011 cohort of 2047 men and women aged 50-69 years. Self-reported measures included ACEs (Centre for Disease Control ACE questionnaire), PSS (Oslo Social Support Scale) and depressive symptoms (CES-D). The primary exposure was self-report of at least one ACE. We also investigated the effects of ACE exposure by ACE scores and ACE subtypes abuse, neglect and household dysfunction. Associations between each of these exposures and depressive symptoms were estimated using logistic regression, adjusted for socio-demographic factors. We tested whether the estimated associations varied across levels of PSS (poor, moderate and strong).

Results $23.7 \%$ of participants reported at least one ACE $(95 \% \mathrm{Cl} 21.9 \%$ to $25.6 \%)$. ACE exposures (overall, subtype or ACE scores) were associated with a higher odds of depressive symptoms, but only among individuals with poor PSS. Exposure to any ACE (vs none) was associated with almost three times the odds of depressive symptoms (adjusted OR 2.85; 95\% Cl 1.64 to 4.95 ) among individuals reporting poor PSS, while among those reporting moderate and strong PSS, the adjusted ORs were 2.21 (95\% $\mathrm{Cl} 1.52$ to 3.22$)$ and 1.39 (95\% Cl 0.85 to 2.29$)$, respectively. This pattern of results was similar when exposures were based on ACE subtype and ACE scores, though the interaction was clearly strongest among those reporting abuse.

Conclusions ACEs are common among older adults in Ireland and are associated with higher odds of later-life depressive symptoms, particularly among those with poor PSS. Interventions that enhance social support, or possibly perceptions of social support, may help reduce the burden of depression in older populations with ACE exposure, particularly in those reporting abuse.

\section{INTRODUCTION}

A life-course approach to mental health views mental illness as a product of biological and social factors that operate across the lifespan. ${ }^{1}$ The stress sensitisation theory ${ }^{2}$ suggests that
Strengths and limitations of this study

A wide range of demographic and health information collected using validated, standardised instruments and questionnaires.

- Large sample size ( $\mathrm{n}=2047)$, study population is representative of the source population reported in national census data.

- Assessment of 10 types of adverse childhood experiences (ACEs) under three ACE subtypes, that is, abuse, neglect household dysfunction.

- Informs future interventions seeking to prevent or manage mental ill-health among those with ACE exposure.

Risk of recall bias from retrospective self-report.

childhood adversity reduces an individual's threshold for developing depressive reactions towards stressful events, causing one to have depressive reactions towards current mild stressors or greater reactivity towards severe stressful events. For example, young women who were exposed to childhood adversities such as domestic violence, parent psychopathology and alcoholism are at a higher risk for depression following exposure to mild stress than women without a history of adversity. ${ }^{3}$ Women with a history of childhood abuse have higher adrenocorticotropic hormone (ACTH), cortisol and heart rate responses to psychosocial stress such as public speaking compared with those without a history of childhood abuse. ${ }^{4}$ The relationship persists into older adulthood; data from the Health and Retirement Study, a US population-based study of adults age $50+$, showed that in accordance with the stress sensitisation theory, childhood trauma (especially physical abuse) amplifies the effect of stresses in adulthood on depressive symptoms. ${ }^{5}$

These psychosocial and neurobiological findings converge on the idea that early-life adversities have an enduring effect on how 
one responds to stressful life events, hence, setting the life-course trajectory for one's mental health.

Adverse childhood experiences (ACEs) encompass any acts of commission or omission by a parent or other caregiver that result in harm, potential for harm or threat of harm to a child in the first 18 years of life, even if harm is not the intended result. ${ }^{6}$ While the association between ACEs and poor mental health has been reported previously, ${ }^{7-10}$ there remains a lack of research on factors that may modify this relationship. Identifying factors that alter the processing of stressful events following exposure to ACEs may be a valuable tool in developing interventions aimed at preventing or mitigating the long-term mental health consequences of ACEs. ${ }^{11}$

Social support may have a protective or buffering effect against the consequences of a stressful event by enhancing cognitive and emotional processing of the experience. ${ }^{12} 13$ This facilitates reappraisal of the stressful event in a manner that is psychologically adaptive. ${ }^{12}{ }^{13}$ Findings from meta-analyses have found a lack of social support to be the single strongest predictor of post-traumatic stress symptoms in both military and civilian populations with a history of psychological trauma. ${ }^{12}{ }^{14}$ The term social support encompasses perceived and received support. It is suggested that perceived support is best understood as an individual difference variable, with evidence that those who report that others will provide them with aid when they are in need (perceived social support (PSS)) are protected from the pathogenic effects of life stress. ${ }^{15}$ Studies on PSS have consistently shown it to be associated with reduced stress and improved physical and mental health, ${ }^{16}$ and that perception of available social support was found to be a better buffer of psychological distress than actual availability of social support in some studies. ${ }^{12} 17$ This suggests that enhancing perception of available support may be just as important, if not more, than increasing actual social support in interventions aimed at moderating psychological effects of stress. Although a number of studies have generated findings supportive of the role of PSS, most of these studies were focused on female victims of childhood sexual abuse. ${ }^{18} 19$ Therefore, PSS is a potentially modifiable risk factor, with evidence to show that social support interventions are associated with improvements in measures of quality of life and burden of illness. ${ }^{20}$

This present study aims to build on prior research by examining whether three ACE subtypes (abuse, neglect and household dysfunction) are related to later-life depressive symptoms, and if so, whether these associations vary across levels of PSS. In line with recent work that suggests that multiple ACEs have an increasingly greater effect on mental health, this study also aims to examine the association between ACE scores and depressive symptoms and if PSS differentially impacts depressive symptoms across an accumulation of ACEs.

\section{METHOD}

\section{Study design and population}

Our analysis uses baseline data from the Mitchelstown cohort, ${ }^{21}$ a study of 50-69-year-old adults randomly selected from patients attending the Livinghealth Clinic in Mitchelstown, Ireland, in 2010-2011. The study population is representative of the profile of the source population reported in national census data. ${ }^{21} \mathrm{~A}$ complete description of the study was sent out to all selected participants with a reply slip indicating acceptance or refusal. After written, informed consent was obtained, the participants completed a detailed health and lifestyle questionnaire and attended a physical examination conducted by research nurses using standardised and validated instruments. Participants were offered separate sealed envelopes to submit their responses to the ACE questionnaire during data collection. Ethical approval for the original study was granted by the Clinical Research Ethics Committee of the Cork Teaching Hospitals.

\section{Predictors}

\section{Adverse childhood experiences}

Exposure to ACE was assessed using the ACE questionnaire ${ }^{2223}$ which addresses 10 individual ACEs under three categories:

- abuse: emotional, physical and sexual abuse

- neglect: emotional and physical neglect

- household dysfunction: parental separation/divorce, violence against mother, household substance abuse, household mental illness and incarceration of household member.

The ACE questionnaire is a reliable and valid measure of childhood adversity that has been used extensively in large-scale ACE studies. ${ }^{22} 23$

All questions about ACEs pertained to the respondents' first 18 years of life and were binary (yes vs no). From these, a dichotomous variable was created to reflect exposure to any ACE, and similar variables were created to reflect any exposure to each ACE subtype (abuse, neglect, household dysfunction). We also calculated a total ACE score for each participant $(+1$ for each of the 10 types of ACE reported). ACE scores of $\geq 3$ were combined into one category due to small sample sizes in some strata, and the score was treated as a categorical variable $(0,1,2$ or $\geq 3)$ to capture any potential non-linearities in the relationship with depressive symptoms. This method of ACE score categorisation has been used previously. ${ }^{24} 25$

\section{Perceived social support}

PSS was assessed using the self-administered Oslo Social Support Scale with three questions ${ }^{26}$ :

- Oslo 1: How many people are you so close to that you can count on them if you have great personal problems? (none (1), 1-2 (2), 3-5 (3), 5+ (4))

- Oslo 2: How much interest and concern do people show in what you do? (a lot (5), some (4), uncertain (3), little (2), none (1)) 
- Oslo 3: How easy is it to get practical help from neighbours if you should need it? (very easy (5), easy (4), possible (3), difficult (2), very difficult (1))

The response categories were assessed independently for each of the three questions, and a sum score was created by summarising the three scores. The Oslo Social Support Scale has been used in several studies, thus supporting its feasibility and predictive validity with respect to psychological distress. ${ }^{27} 28$ A sum score ranging between 3 and 8 was categorised as poor, a score between 9 and 11 as moderate and a score of $12-14$ as strong PSS. ${ }^{26}$ This categorisation was used previously in a study assessing PSS in a population of older adults. ${ }^{29}$

\section{Outcomes}

\section{Depressive symptoms}

The CES-D questionnaire ${ }^{30}$ was used to assess for depressive symptoms. The items of the scale are symptoms associated with depression which have been used in longer, previously validated instruments, and have been tested in both household and clinical settings. ${ }^{30}$ It has very high internal consistency and adequate test-retest repeatability. ${ }^{30}$

The score is a sum of 20 questions. The possible range of scores is $0-60$, with higher scores indicating the presence of greater symptomatology. A score between 16 and 21 on the CES-D scale indicates the presence of mild to moderate depressive symptoms while a score of $\geq 22$ indicates the possibility of major depression. ${ }^{30}$ Participants with a score of $\geq 16$ were defined as having depressive symptoms. ${ }^{30}$ This cut-off point has been used extensively in other studies in identifying individuals at risk of clinical depression. ${ }^{31} 32$

\section{Covariates}

Educational attainment was ascertained by the question 'What is the highest level of education you have completed?' and responses were categorised into primary, secondary or tertiary level.

Current marital status was ascertained by the question 'What is your current marital status?' and the options were single (never married), separated, cohabiting, divorced, married or widowed.

Participants were asked whether they were covered by the General Medical Services (GMS) scheme, which entitles those covered to free medical care at the point of access. Responses were categorised as GMS patient (yes/ no). GMS eligibility is based on low-income thresholds.

Smoking status was categorised as never smoked, current smoker or former smoker in response to the questions 'Have you smoked at least 100 cigarettes in your entire life?' and 'Are you a current smoker?'

Alcohol consumption was derived from the question 'During the past 7 days how many standard drinks of any alcoholic beverage did you have each day?' and was categorised as non-drinker ( $<1$ unit/week), moderate drinker (1-14 units/week) and heavy drinker (>14 units/week). ${ }^{33}$
Physical activity was measured as metabolic equivalents (METs) minutes per week using the short-form International Physical Activity Questionnaire ${ }^{34}$ and was categorised into three groups (low, moderate or high) based on MET minutes per week in all activity types.

Height and weight were measured using standardised methods by study personnel and used to calculate body mass index (BMI, $\mathrm{kg} / \mathrm{m}^{2}$ ). Participants were classified as underweight if their BMI was $<18.5 \mathrm{~kg} / \mathrm{m}^{2}$, normal if 18.5 to $<25 \mathrm{~kg} / \mathrm{m}^{2}$, overweight if 25 to $<30 \mathrm{~kg} / \mathrm{m}^{2}$ and obese if $\geq 30 \mathrm{~kg} / \mathrm{m}^{2}$.

\section{Statistical methods}

Descriptive data and crude tests of association

Continuous variables were described by means and SD. Categorical variables were described by counts and percentages. Student's t-test, one-way analysis of variance or Pearson's $\chi^{2}$ test were used as appropriate to test for differences in the distributions of demographic, health and lifestyle measures between the groups with and without ACE.

\section{Logistic regression}

Associations between each ACE exposure (any ACE, each ACE subtype and ACE score; and in a set of supplemental models, each of the 10 individual ACEs) and depressive symptoms were estimated using logistic regression in two models, A and B. Model A included the main effects of the ACE exposure, PSS and a product interaction term between the two. Model B included additional effects to adjust for potential confounding and selection biases. These were age, gender and educational attainment, current marital status and GMS cover. Results were reported as ORs with 95\% CIs. Interaction terms were tested using type II sums of squares likelihood ratio $\chi^{2}$ test (LRT), and exact $\mathrm{p}$ values were reported.

\section{Quantile regression}

Our main analyses use categorisations of the CES-D and the Oslo Social Support Scale to define depressive symptoms (yes/no) and PSS (low/medium/strong). While these categorisations are not uncommon, there will be some loss of power associated with their use. We thus used quantile regression ${ }^{35}$ in a supplemental analysis to model the association between CES-D scores and ACE (any ACE, any abuse, any neglect or any household dysfunction), conditional on the selected covariates. Quantile regression is analogous to multiple linear regression, except that it models a given centile of the outcome's distribution, the median in our case, rather than the outcome's mean. It is thus robust to departures from the normally distributed errors assumption of linear regression, which is relevant given the skewed distribution of CES-D scores in our sample.

\section{Missing data}

Missing data were handled using multiple imputation, so that all participants who completed the baseline questionnaire were included in the analytical sample, even if they 
were missing values for one or more variables. For each estimated statistical model, 30 imputed data sets were created, after a burn-in of 30 replications using predicted mean matching. ${ }^{36}$ Each imputation model included all variables used in a given statistical model, allowed for non-linear relationships using restricted cubic splines with three knots, and included the key interaction of interest $(\mathrm{ACE} \times \mathrm{PSS})$. The statistical model of interest was then estimated in each imputed data set, and parameter estimates were combined using Rubin's rules. ${ }^{37} \mathrm{~A}$ complete case sensitivity analysis was also performed for comparison.

\section{Inference}

All parameter estimates are reported with 95\% CIs and/ or exact $p$ values. While we have estimated a fairly large number of parameters, we have not selectively reported any of these, nor made any other decisions based on statistical significance testing. This is consistent with current practice in major epidemiological journals, particularly with observational study designs, and recent guidance from the American Statistical Association. ${ }^{38}$

All analyses were conducted using the $\mathrm{R}$ Project for Statistical Computing (V.3.3.1) ${ }^{39}$

\section{Research reporting}

The Strengthening the Reporting of Observational Studies in Epidemiology guidelines were used to inform the study report.

\section{RESULTS}

\section{Baseline characteristics}

Of the 3051 people invited to participate in this study, $2047(67 \%)$ completed the baseline assessment. The mean age at baseline was 55.8 years and $51 \%$ of the participants were female.

Of these, $1926(94 \%)$ completed the ACE questionnaire. $23.7 \%$ ( $\mathrm{n}=457 ; 95 \% \mathrm{CI} 21.9$ to 25.6$)$ of the respondents reported at least one form of ACE. 16.1\% ( $\mathrm{n}=302$, 95\% CI 14.4 to 17.7) of the participants reported depressive symptoms.

The characteristics of respondents with and without self-reported ACEs are summarised in table 1. Prevalence of participants with a CES-D score indicative of major depression was significantly higher among participants who reported ACE compared with participants who did not $(14.1 \%$ vs $6.0 \%, \mathrm{p} \leq 0.001)$. Prevalence of poor PSS was also higher among participants who reported ACEs (19.6\% vs $10.7 \%, \mathrm{p} \leq 0.001)$. Participants who reported ACEs tended to be younger, separated/divorced, have GMS cover, reported long-term illness/disability and had attained tertiary education.

\section{Logistic regression}

Table 2 gives ORs and 95\% CIs for models A and B where any $A C E$ was the exposure variable. Exposure to any ACE was associated with almost three times the odds for depressive symptoms among participants reporting low PSS (adjusted OR 2.85, 95\% CI 1.64 to 4.95 ). The association between any ACE and depressive symptoms was substantially attenuated with moderate PSS (OR 2.21, 95\% CI 1.52 to 3.22 ) and strong PSS (OR 1.39, 95\% CI 0.85 to 2.29 ) (figure 1 ). The LRT $\mathrm{p}$ value for the interaction term was 0.19 in the full adjusted model. The estimates were similar in the unadjusted and adjusted models (table 2), as were results from the complete case analysis (online supplementary table 1 ).

Similarly, each ACE subtype was also associated with increased odds of depressive symptoms among individuals reporting low PSS, and this association was reduced among individuals reporting moderate and high PSS (figure 1 and online supplemental tables 2-4). The odds of depressive symptoms among those reporting abuse (vs not) were more than five times greater in individuals reporting low PSS (OR 5.20, 95\% CI 2.71 to 9.99), three times greater in those reporting moderate PSS (OR $3.22,95 \%$ CI 2.11 to 4.92 ), but only slightly increased in those reporting high PSS (OR 1.29, 95\% CI 0.68 to 2.45). Among those reporting neglect, those figures were OR 3.31 (95\% CI 1.76 to 6.20 ), OR 3.31 (95\% CI 1.63 to 5.73 ) and 1.81 (95\% CI 0.83 to 3.95 ), respectively; and among those reporting household dysfunction, the estimates were OR 2.24 (95\% CI 1.24 to 4.06), OR 1.87 (95\% CI 1.20 to 2.90 ) and OR 1.24 (95\% CI 0.68 to 2.28$)$.

The odds of depressive symptoms were progressively higher among individuals who experienced a greater ACE score (figure 2 and online supplementary table 5). These associations were again strongest in those reporting poor PSS, for whom an ACE score of 1 (vs 0 ) was associated with 1.44 times the odds of depressive symptoms (95\% CI 0.61 to 3.37), the OR for an ACE score of 2 was 3.08 (95\% CI 1.24 to 7.65) and the OR for an ACE score of 3+ was 5.33 (95\% CI 2.56 to 11.10). Overall, evidence for effect modification of ACE exposures by PSS was strongest for abuse, with an LRT p value of 0.011 (online supplementary table 2), while it was 0.16 and 0.23 for neglect and household dysfunction, respectively (online supplementary tables 3 and 4 ).

Regarding individual ACEs, those reflecting abuse (online supplementary table 6) and neglect (online supplementary table 7) tended to be more strongly associated with depressive symptoms than those reflecting the various forms of household dysfunction (online supplementary table 8). Further, the effect modification by PSS was most clearly demonstrated for the estimated effects of abuse.

The quantile regression model for continuously measured CES-D scores gave qualitatively similar estimated to the logistic regression models (online supplementary table 9): any ACE, any abuse, any neglect and any household dysfunction were each associated with higher median CES-D scores, as was lower PSS score. Further, the association between ACE and CES-D score was highest in those with lower PSS scores. 
Table 1 Baseline characteristics of participants in the Mitchelstown cohort study, 2010-2011, stratified by self-reported adverse childhood experience (ACE) ( $n=1926)$

\begin{tabular}{|c|c|c|c|c|c|c|}
\hline \multirow[b]{2}{*}{ Characteristics } & $\begin{array}{c}\text { With ACE } \\
(n=457 ; 23.7 \%)\end{array}$ & $\begin{array}{c}\text { Without ACE } \\
(n=1469 ; 76.3 \%)\end{array}$ & \multicolumn{3}{|c|}{ Group difference } & \multirow{2}{*}{$\begin{array}{c}\begin{array}{c}\text { Missing } \\
\text { data }\end{array} \\
\mathbf{N}(\%) \\
\end{array}$} \\
\hline & \multicolumn{2}{|c|}{ Estimate $^{*}$} & Test statistic† & df & p Value & \\
\hline Age (years) & $53.6(16.9)$ & $56.6(15.2)$ & 3.54 & 1924 & $\leq 0.001$ & 0 \\
\hline \multicolumn{7}{|l|}{ Sex } \\
\hline Male & 234 (51.2) & 707 (48.1) & 1.32 & 1 & 0.25 & 0 \\
\hline Female & $223(48.8)$ & $762(51.9)$ & & & & \\
\hline \multicolumn{7}{|l|}{ Marital status } \\
\hline Single (never married) & $45(10.0)$ & $116(8.0)$ & 28.85 & 3 & $\leq 0.001$ & $18(0.9)$ \\
\hline Cohabiting/married & 334 (74.2) & $1175(80.6)$ & & & & \\
\hline Separated/divorced & $52(11.6)$ & $73(5.0)$ & & & & \\
\hline Widowed & $19(4.2)$ & $94(6.4)$ & & & & \\
\hline \multicolumn{7}{|l|}{ Perceived social support } \\
\hline Poor & $86(19.8)$ & $148(10.6)$ & 27.10 & 2 & $\leq 0.001$ & $101(5.2)$ \\
\hline Moderate & $193(44.5)$ & $632(45.4)$ & & & & \\
\hline Strong & $155(35.7)$ & $611(43.9)$ & & & & \\
\hline \multicolumn{7}{|l|}{ Socioeconomic } \\
\hline \multicolumn{7}{|l|}{ Education } \\
\hline Primary & $116(26.9)$ & $382(27.7)$ & 10.57 & 2 & 0.01 & $117(6.1)$ \\
\hline Secondary & $192(44.4)$ & $701(50.9)$ & & & & \\
\hline Tertiary & $124(28.7)$ & $294(21.4)$ & & & & \\
\hline \multicolumn{7}{|l|}{$\begin{array}{l}\text { General Medical Services } \\
\text { cover }\end{array}$} \\
\hline Yes & $158(34.6)$ & $432(29.4)$ & 4.31 & 1 & 0.04 & 0 \\
\hline No & $299(65.4)$ & $1037(70.6)$ & & & & \\
\hline \multicolumn{7}{|l|}{ Personal health behaviours } \\
\hline \multicolumn{7}{|l|}{ Smoking } \\
\hline Never smoked & $200(45.0)$ & $750(52.9)$ & 8.93 & 2 & 0.01 & $64(3.3)$ \\
\hline Former smoker & $175(39.4)$ & $462(32.6)$ & & & & \\
\hline Current smoker & $69(15.5)$ & $206(14.5)$ & & & & \\
\hline \multicolumn{7}{|l|}{ Alcohol } \\
\hline Non-drinker & $56(17.7)$ & $202(21.4)$ & 3.07 & 2 & 0.22 & $667(34.6)$ \\
\hline Moderate drinker & 208 (65.6) & $610(64.8)$ & & & & \\
\hline Heavy drinker & $53(16.7)$ & $130(13.8)$ & & & & \\
\hline \multicolumn{7}{|l|}{ Physical activity } \\
\hline Low & $211(49.8)$ & $667(47.7)$ & 0.70 & 2 & 0.70 & $104(5.4)$ \\
\hline Moderate & $120(28.3)$ & $423(30.3)$ & & & & \\
\hline High & 93 (21.9) & $308(22.0)$ & & & & \\
\hline \multicolumn{7}{|l|}{ Personal health history } \\
\hline \multicolumn{7}{|l|}{ Self-rated health status } \\
\hline Very good & $123(27.2)$ & $435(30.1)$ & 12.06 & 4 & 0.02 & $30(1.5)$ \\
\hline Good & $235(52.0)$ & $804(55.7)$ & & & & \\
\hline Fair & $81(17.9)$ & $178(12.3)$ & & & & \\
\hline Poor & $11(2.4)$ & $20(1.4)$ & & & & \\
\hline Very poor & $2(0.4)$ & $7(0.5)$ & & & & \\
\hline
\end{tabular}


Table 1 Continued

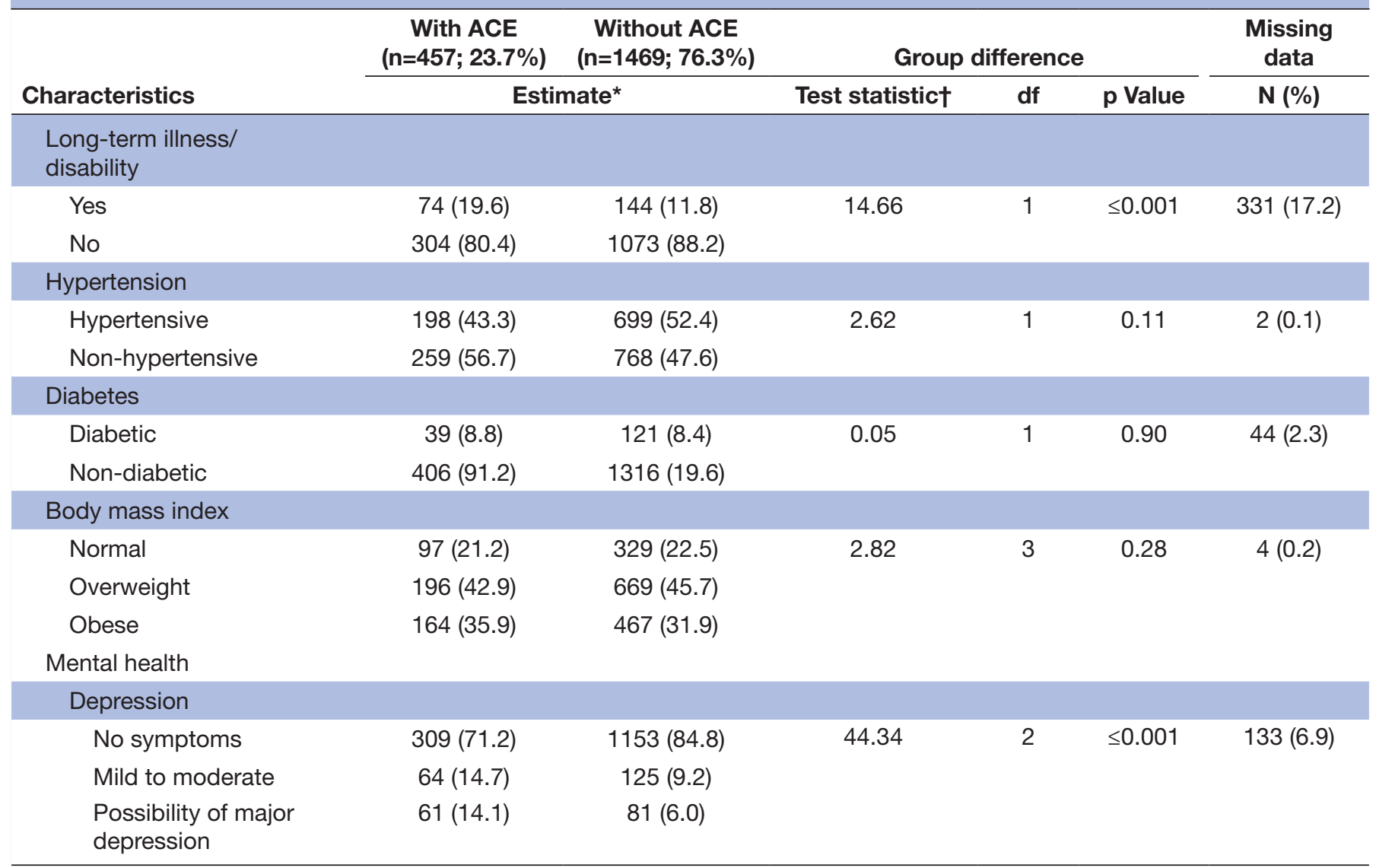

${ }^{\star}$ Estimates are reported as mean (SD) for continuous variables; and $\mathrm{N}(\%)$ for categorical variables.

†Differences in means were tested using Student's t-test) ${ }^{54}$; dependence between categorical variables was tested using Pearson's $\chi^{2}$ test ${ }^{55}$.

\section{DISCUSSION}

In this population-based study, exposure to ACEs and reporting poor social support were both related to a higher odds of depressive symptoms later in life, even after controlling for demographic and socioeconomic factors. While other studies have reported an association between ACE and later-life depression, ${ }^{40-42}$ few have explored the role of PSS as a potential effect modifier. Importantly, we found that the deleterious impact of ACEs was typically limited to those individuals who also reported poor and moderate PSS. However, the statistical evidence for this interaction was only strong among those reporting abuse.

In this sample, $23.7 \%$ reported having experienced at least one form of ACE. This is low compared with international and national estimates. The prevalence of self-reported ACE was $\sim 66 \%$ in the ACE study, ${ }^{23}$ a collaboration between the Centers for Disease Contol and Prevention (CDC) and Kaiser Permanente, while in the Irish Longitudinal Study of Ageing (TILDA), the prevalence was $33.6 \%{ }^{43}$ The higher prevalence of ACE in TILDA may be explained by the broader nature of the ACE measure. In contrast to the 10-item ACE questionnaire used in the current study, a 4-item measure was used in TILDA to capture socioeconomic disadvantage, parental substance abuse, physical abuse and sexual abuse. ${ }^{43}$

Prevalence of depressive symptoms and poor PSS were substantially higher among participants who reported ACE compared with those who did not. Perception of social support level did not significantly differ between men and women in our study, contrary to literature suggesting that women tend to perceive their social support as stronger than men do. ${ }^{44}$ Prevalence of separation/divorce, GMS cover and long-term illness/disability was found to be markedly higher among participants who reported ACEs. These findings suggest that exposure to childhood adversity could affect a multitude of factors across the lifespan. Animal studies have shown that early stressors result in long-term changes in oxytocin, a peptide that regulates pair bonding and social attachment. ${ }^{45}{ }^{46}$ It is possible that this may extend to individuals with ACEs, accounting for their impaired ability in forming long-term social attachments, but it is important to note that our study does not speak directly to this speculation.

Exposure to all ACE subtypes among those who perceived poor social support was significantly related to higher odds for later-life depression. Though the estimated impact on depressive symptoms was strongest for abuse, the less severe forms of childhood adversity such 
Table 2 Logistic regression analyses for adverse childhood experience (ACE) and later-life depression ( $\left.n=2047^{\star}\right)$

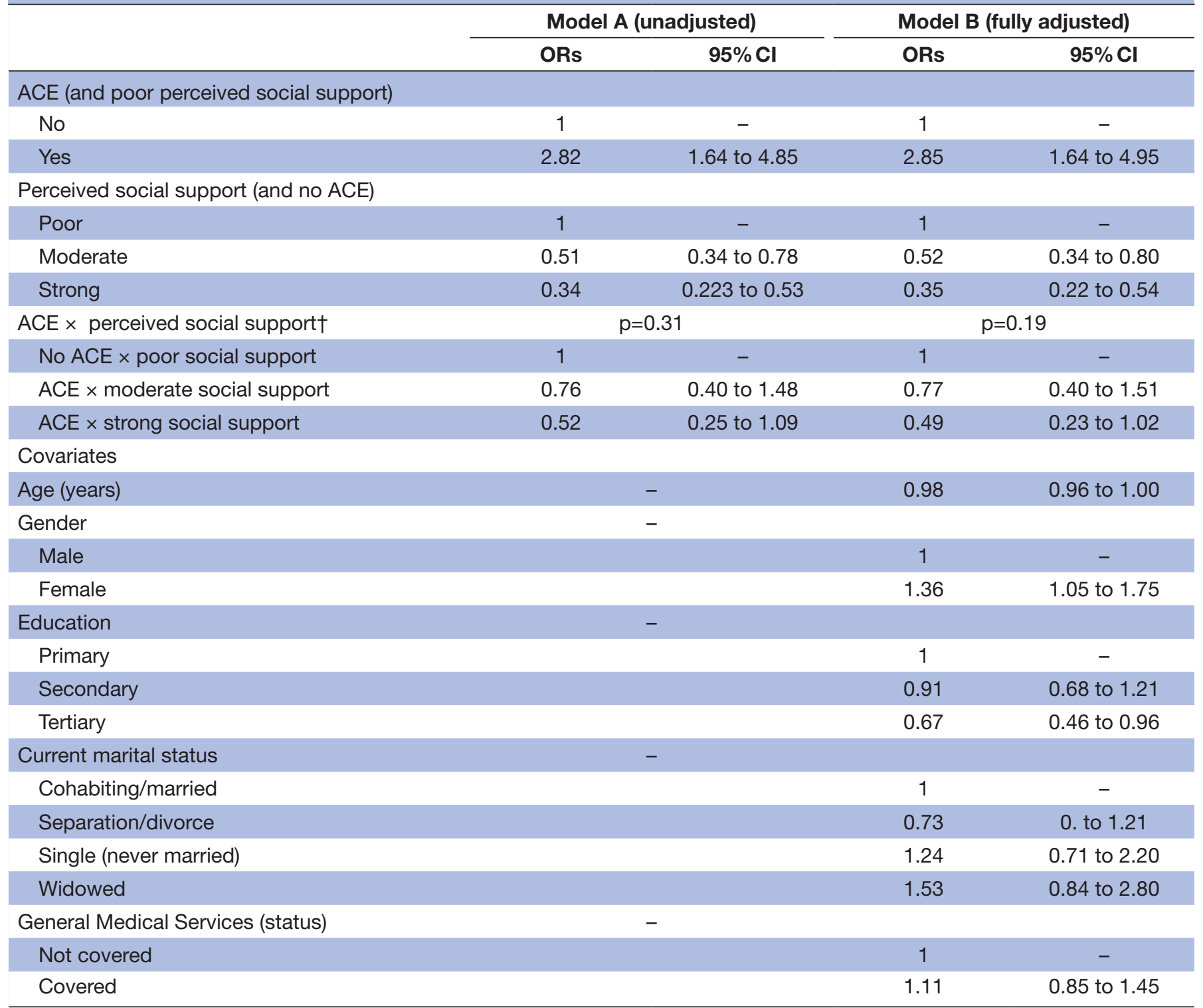

${ }^{*}$ Parameter estimates based on the complete sample with missing data accounted for with multiple imputation with predictive mean matching.

$\mathrm{tp}$ values are from the type II sum of squares likelihood ratio $\chi^{2}$ test for the interaction term.

as neglect and household dysfunction may also have longterm effects on mental health. Further, the experience of any number of childhood adversity, from having experienced one type of ACE to three or more types of ACE, is associated with increased odds for later-life depression, in the presence of poor PSS.

Consistent with the stress buffering model, ${ }^{17}$ the relationship between ACE (overall, subtype or ACE score) and depression later in life was found to vary according to level of PSS, though this interaction was clearly strongest among those reporting abuse. These findings highlight that the strengthening of social support among childhood adversity survivors may benefit mental health.

Social support may enhance cognitive and emotional processing of the experience, hence, facilitating reappraisal of the stressful event in a manner that is psychologically adaptive. ${ }^{12} 13$ Findings from experience sampling method studies highlight the role of positive emotional content in buffering negative reactivity of stress ${ }^{47}$ and in improving responsiveness towards antidepressant medications. ${ }^{48}$ These findings suggest that in addition to preventing depression improving social support may serve as an important adjunct in the medical management of depression. The role of social support may be especially important for older persons as this is a phase of major social transitions such as retirement and bereavement. ${ }^{49}$

There is also evidence for the role of PSS among those with chronic medical conditions. Higher levels of PSS have been associated with longer survival following heart attacks ${ }^{50}$ and improved well-being, that is, mental health, perceived burden of illness and 


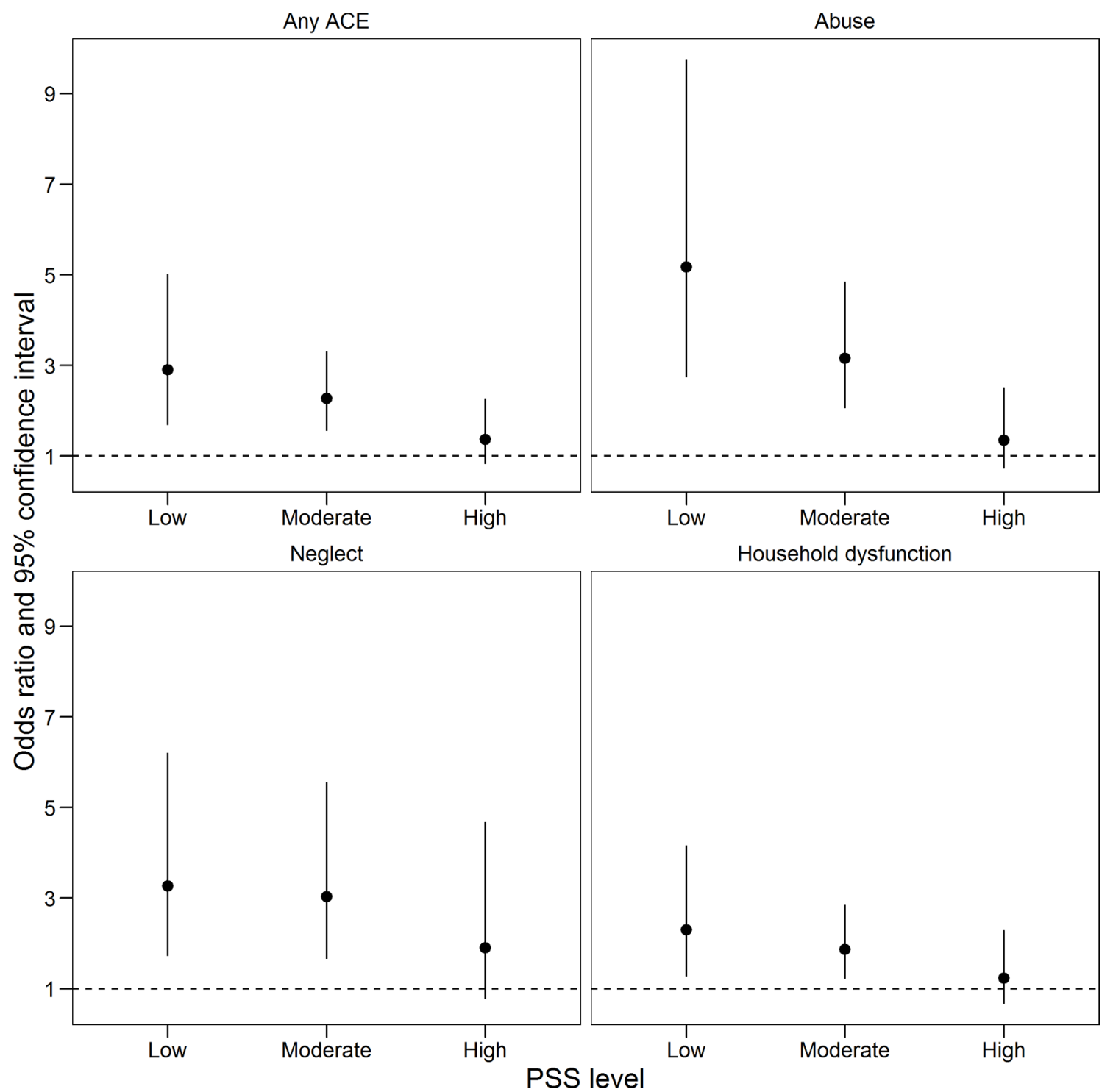

Figure 1 ORs and 95\% Cls from a logistic regression model of later-life depressive symptoms and any adverse childhood experience (ACE) or ACE subtypes, illustrating the interaction with perceived social support (PSS) $(n=2047)$.

quality of life among patients with end-stage renal disease. ${ }^{20}$

\section{Strengths and limitations}

Demographic information and personal histories in this study were obtained using validated, standardised instruments and questionnaires of health and well-being. ${ }^{21}$ Although the sample is a relatively homogenous, Caucasian population taken from a single, large primary care centre, it is representative of the profile of the source population reported in national census data. ${ }^{21}$

ACE was measured by retrospective self-report of events that happened $\sim 30$ years previously. There may be a risk of recall bias due to the time lapse between the events in question and the survey. Questions that concerned less objective events such as whether the participant felt unloved may also be subject to greater recall bias and individual interpretation. Despite the risk of recall bias, the ACE questionnaire has been previously shown to have good test-retest reliability. ${ }^{40}$ The sensitive nature of the questions and the participant's perception of the 'social taboos' of responding to such questions may also be an important limitation. This was acknowledged during data collection by offering patients a separate sealed envelope in which to submit their responses.

We have reported models for each of the 10 individual ACEs. However, given the relatively small number of participants experiencing any one specific ACE, the respective parameter estimates will be volatile. While those results qualitatively conformed with the models for any ACE and ACE subtypes, a larger study would be needed to further examine the impact of the individual ACEs.

There was a non-negligible amount of missing data, which is not uncommon for such studies. We have used multiple imputation, rather than case-wise deletion, to both improve the efficiency of analyses (by retaining more observations in the analysis) and to reduce chances 


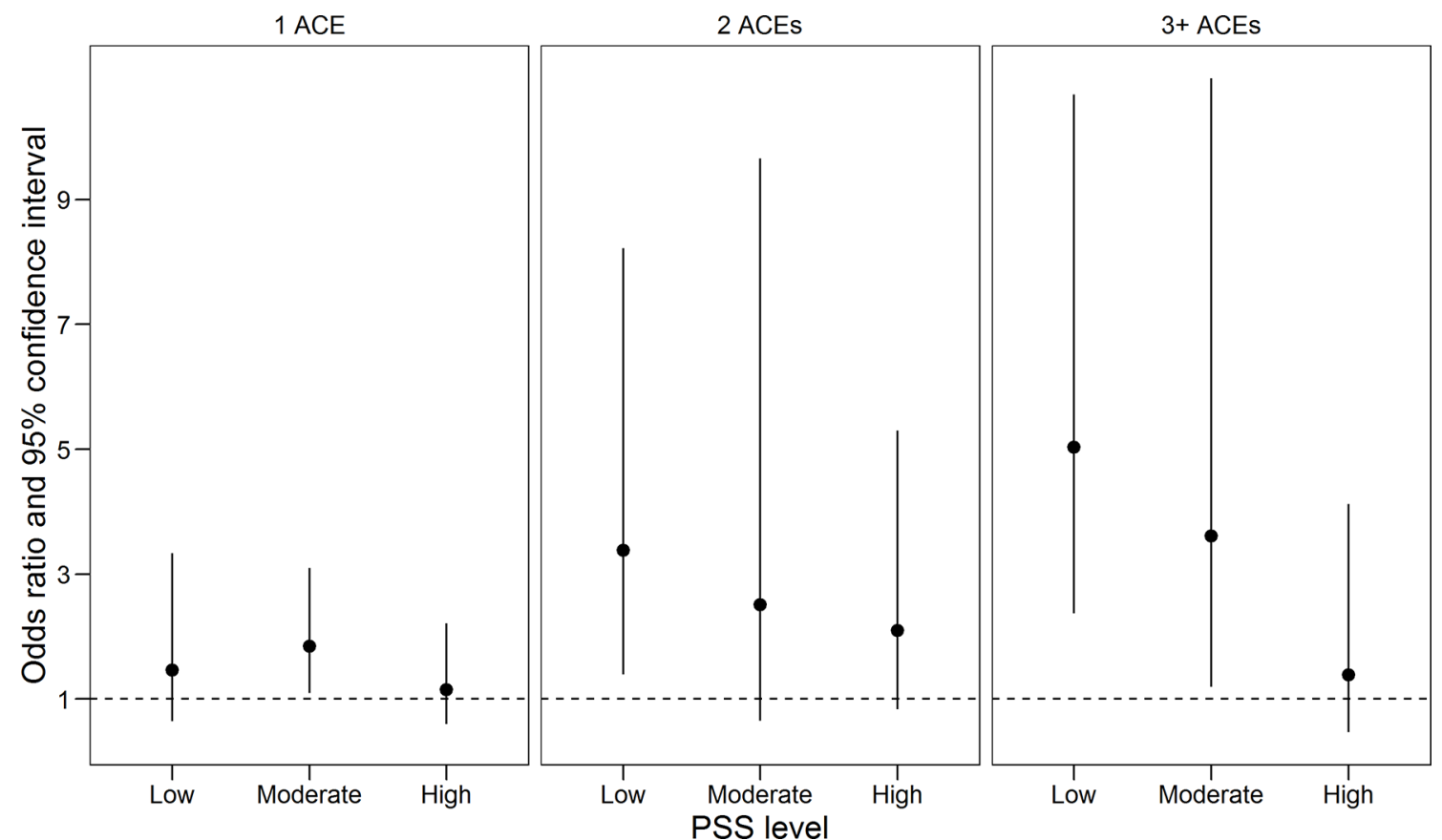

Figure 2 ORs and 95\% Cls from a logistic regression model of later-life depressive symptoms and adverse childhood experience (ACE) score, illustrating the interaction with perceived social support (PSS) ( $n=2047)$.

of bias. Multiple imputation, assuming the model was correctly specified, is unbiased given an assumption that data were missing at random, conditional on other variables accounted for in the model (ie, the Missing at random (MAR) assumption). This is a more defensible position than the assumption that data were missing completely at random required for valid estimates using case-wise deletion. However, we cannot rule out the possibility that missing data, particularly for ACEs and the CES-D, were missing not-at-random.

Lastly, and most importantly, this is an observational study where both the exposure and outcomes will certainly share causes. We have tried to adjust for this through the careful selection and adjustment for confounders, but these in turn will be measured with some error and will certainly not represent an optimal set of covariates to adjust for, so it is important that these results are viewed as part of a larger and still developing body of research.

\section{Implications of findings and further research}

There is evidence that shows the efficacy of social support intervention in improving PSS and psychological distress symptoms, specifically among women who had experienced intimate partner violence. ${ }^{51}$ This intervention was led by trained nurses and founded on the four modalities of social support, that is, belonging, evaluation, self-esteem and tangible support. ${ }^{51}$ Belonging was done through listening and responding to others who had experienced intimate partner violence. ${ }^{51}$ Evaluation involved helping women see themselves as others see them. ${ }^{51}$ Self-esteem was promoted by focusing on their strengths and achievements in surviving domestic violence. ${ }^{51}$ Tangible support involved discussions of resources in the community for help they need such as financial assistance and healthcare. ${ }^{51}$

Results of our study have potential implications for clinicians seeking to prevent mental illness among survivors of childhood adversity. Interventions that aim to protect mental health among survivors of childhood adversity might benefit from strengthening social support or perhaps even just perception of social support. There is an increasing literature showing the positive effect of interventions that increase perceptions of social support in patients with terminal disease or end-stage kidney disease. ${ }^{20}$ By showing the buffering effect of PSS on the ACE-mental health relationship, our findings highlight the potential for interventions targeting PSS to reduce the likelihood of depression in patients who experienced childhood adversity. Individuals with a history of childhood adversity may experience deficits in support-seeking behaviour and social attachments. Hence, interventions may include social skills training where participants are equipped with skills to identify, invite and maintain healthy social connections with others. ${ }^{12}{ }^{52}$ Further research on the implementation and efficacy of such interventions is indicated.

In addition, our findings show that the contribution of ACEs to mental health impairment persists across the life course. Despite such findings, ACE screening is not routinely undertaken in clinical practice. Twenty-five per cent of primary care physicians in the Massachusetts Academy of Family Physicians reported that they never or rarely screened for childhood trauma in adult patients. ${ }^{53}$ Further work on how this can be implemented effectively in primary care and medical training is urgently needed.

Previous presentations 
1. Medicine and the Humanities and Social Sciences Conference, Sam Houston State University, Huntsville, Texas, USA; 4-5 March 2015.

2. The Atlantic Medical Corridor Conference, University College Cork, Western Road, Cork, Ireland; 10 November 2014.

3. The 18th International Conference on Public Health, The World Academy of Science, Engineering and Technology, London; 23-24 May 2016.

Acknowledgements The authors thank the participants, study nurses, administrators and clinical staff at the Livinghealth Clinic, Mitchelstown, Cork, Ireland

Contributors PMK conceived of the study. PMK, DD and CS provided statistical expertise in the study design. EVC conducted the secondary data analysis and interpretation and drafted the manuscript. All authors contributed to critical revision of the article and approved the final manuscript.

Funding The Mitchelstown cohort study is supported by a research grant from the Irish Health Research Board (reference HRC/2007/13).

Competing interests Health Research Board, Ireland (SSS'2014'781 to EVC). DD was further supported by a HRB Interdisciplinary Capacity Award (ICE/2012/12). CS was supported by the HRB National SpR Academic Fellowship Award (NSAFP/2011/3).

Patient consent Obtained.

Ethics approval Clinical Research Ethics Committee of the Cork Teaching Hospitals.

Provenance and peer review Not commissioned; externally peer reviewed.

Data sharing statement № additional data are available.

Open Access This is an Open Access article distributed in accordance with the Creative Commons Attribution Non Commercial (CC BY-NC 4.0) license, which permits others to distribute, remix, adapt, build upon this work non-commercially, and license their derivative works on different terms, provided the original work is properly cited and the use is non-commercial. See: http://creativecommons.org/ licenses/by-nc/4.0/

(C) Article author(s) (or their employer(s) unless otherwise stated in the text of the article) 2017. All rights reserved. No commercial use is permitted unless otherwise expressly granted.

\section{REFERENCES}

1. Brundtland $\mathrm{GH}$. The implications for training of embracing; $A$ life course approach to health. Population 2050;2000:2025.

2. Monroe SM, Simons AD. Diathesis-stress theories in the context of life stress research: implications for the depressive disorders. Psychol Bull 1991;110:406-25.

3. Hammen C, Henry R, Daley SE. Depression and sensitization to stressors among young women as a function of childhood adversity. $J$ Consult Clin Psychol 2000;68:782-7.

4. Heim C, Newport DJ, Heit S, et al. Pituitary-adrenal and autonomic responses to stress in women after sexual and physical abuse in childhood. JAMA 2000;284:592-7.

5. Thalida EM, Arpawong JL, Phillips DF, et al. Prescott. Iongitudinal test of the stress sensitization hypothesis for depression in older age: evaluating the combined impacts of Childhood Trauma and recent stress. Society for Prevention Research 24th annual meeting, 2016. Pacific D/L (Hyatt Regency San Francisco).

6. Gilbert R, Widom CS, Browne K, et al. Burden and consequences of child maltreatment in high-income countries. Lancet 2009;373:68-81.

7. Lansford JE, Dodge KA, Pettit GS, et al. A 12-year prospective study of the long-term effects of early child physical maltreatment on psychological, behavioral, and academic problems in adolescence. Arch Pediatr Adolesc Med 2002;156:824-30.

8. Herrenkohl TI, Herrenkohl RC. Examining the overlap and prediction of multiple forms of Child Maltreatment, Stressors, and socioeconomic status: a longitudinal analysis of Youth Outcomes. $J$ Fam Violence 2007;22:553-62.
9. Banyard VL, Williams LM, Siegel JA. The long-term mental health consequences of child sexual abuse: an exploratory study of the impact of multiple traumas in a sample of women. $J$ Trauma Stress 2001;14:697-715.

10. Thornberry TP, Ireland TO, Smith CA. The importance of timing: the varying impact of childhood and adolescent maltreatment on multiple problem outcomes. Dev Psychopathol 2001;13:957-79.

11. Scott J, Varghese D, McGrath J. As the twig is bent, the tree inclines: adult mental health consequences of childhood adversity. Arch Gen Psychiatry 2010;67:111-2.

12. Evans SE, Steel AL, DiLillo D. Child maltreatment severity and adult trauma symptoms: does perceived social support play a buffering role? Child Abuse Negl 2013;37:934-43.

13. Thoits PA. Social support as coping assistance. J Consult Clin Psychol 1986;54:416-23.

14. Brewin CR, Andrews B, Valentine JD. Meta-analysis of risk factors for posttraumatic stress disorder in trauma-exposed adults. J Consult Clin Psychol 2000;68:748-66.

15. Cohen S. Social relationships and health. Am Psychol 2004;59:676-84.

16. Haber MG, Cohen JL, Lucas T, et al. The relationship between selfreported received and perceived social support: a meta-analytic review. Am J Community Psychol 2007;39-133-44.

17. Cohen S, Wills TA. Stress, social support, and the buffering hypothesis. Psychol Bull 1985;98:310-57.

18. Murthi M, Espelage DL, abuse C. Social support, and psychological outcomes: a loss framework. Child abuse \& neglect 2005;29:1215-31.

19. Hyman SM, Gold SN, Cott MA. Forms of social support that moderate PTSD in childhood sexual abuse survivors. J Fam Violence 2003:18:295-300.

20. Cohen SD. editor Social support interventions will improve the quality of life of ESRD patients. Seminars in dialysis: Wiley Online Library., 2013.

21. Kearney PM, Harrington JM, Mc Carthy VJ, et al. Cohort profile: the Cork and kerry diabetes and heart disease study. Int J Epidemiol 2013;42:1253-62.

22. Anda RF, Butchart A, Felitti VJ, et al. Building a framework for global surveillance of the public health implications of adverse childhood experiences. Am J Prev Med 2010;39:93-8.

23. Centers for Disease Control and Prevention NCfIPaC, Division of Violence Prevention. Adverse Childhood experience study Atlanta2006 . 2014 http://www.cdc.gov/violenceprevention/acestudy/ (accessed 1 Jul 2015).

24. Dube SR, Felitti VJ, Dong M, et al. The impact of adverse childhood experiences on health problems: evidence from four birth cohorts dating back to 1900. Prev Med 2003;37:268-77.

25. Anda RF, Whitfield CL, Felitti VJ, et al. Adverse childhood experiences, alcoholic parents, and later risk of alcoholism and depression. Psychiatr Serv 2002;53:1001-9.

26. Brevik J, Dalgard O. The health profile inventory. Oslo: University of Oslo, 1996.

27. Dalgard OS, Dowrick C, Lehtinen V, et al. ODIN Group. Negative life events, social support and gender difference in depression: a multinational community survey with data from the ODIN study. Soc Psychiatry Psychiatr Epidemiol 2006;41:444-51.

28. Korkeila J, Lehtinen V, Bijl R, et al. Establishing a set of mental health indicators for Europe. Scand J Public Health 2003;31:451-9.

29. Bøen H, Dalgard OS, Bjertness E. The importance of social support in the associations between psychological distress and somatic health problems and socio-economic factors among older adults living at home: a cross sectional study. BMC Geriatr 2012;12:1-12.

30. Radloff LS. The CES-D Scale: a Self-Report Depression Scale for Research in the General Population. Appl Psychol Meas 1977:1:385-401.

31. Lewinsohn PM, Seeley JR, Roberts RE, et al. Center for Epidemiologic Studies Depression Scale (CES-D) as a screening instrument for depression among community-residing older adults. Psychol Aging 1997;12:277-87.

32. Shinar D, Gross CR, Price TR, et al. Screening for depression in stroke patients: the reliability and validity of the Center for Epidemiologic Studies Depression Scale. Stroke 1986;17:241-5.

33. Khaw KT, Wareham N, Bingham S, et al. Combined impact of health behaviours and mortality in men and women: the EPIC-Norfolk prospective population study. PLoS Med 2008;5:376-7.

34. Craig CL, Marshall AL, Sjöström M, et al. International physical activity questionnaire: 12 -country reliability and validity. Med Sci Sports Exerc 2003;35:1381-95.

35. Hallock K, Koenker R. Quantile regression. Journal of Economic Perspectives 2001:15:143-56

36. Van Buuren S. Flexible imputation of missing data: CRC press;, 2012. 
37. Rubin DB. Multiple imputation for Nonresponse in surveys: Harvard Univ, 1987.

38. Wasserstein RL, Lazar NA. The ASA's Statement on $p$-Values: context, process, and purpose. Am Stat 2016;70:129-33.

39. Team RDC. Alanguage and environment for statistical computing. $R$ foundation for statistical computing, Vienna, Austria 2011.

40. Chapman DP, Whitfield CL, Felitti VJ, et al. Adverse childhood experiences and the risk of depressive disorders in adulthood. $J$ Affect Disord 2004;82:217-25.

41. Anda RF, Whitfield CL, Felitti VJ, et al. Adverse childhood experiences, alcoholic parents, and later risk of alcoholism and depression. Psychiatr Serv 2002;53:1001-9.

42. Gilbert R, Widom CS, Browne K, et al. Burden and consequences of child maltreatment in high-income countries. Lancet 2009;373:68-81.

43. McCrory C, Dooley C, Layte R, et al. The lasting legacy of childhood adversity for disease risk in later life. Health Psychol 2015;34:687-96.

44. Cutrona CE. Social support in couples: marriage as a resource in times of stress: Sage Publications;, 1996.

45. Insel TR, Winslow JT. Serotonin and neuropeptides in affiliative behaviors. Biol Psychiatry 1998;44:207-19.

46. Francis DD, Young LJ, Meaney MJ, et al. Naturally occurring differences in maternal care are associated with the expression of oxytocin and vasopressin (V1a) receptors: gender differences. $J$ Neuroendocrinol 2002;14:349-53.
47. Wichers MC, Myin-Germeys I, Jacobs N, et al. Evidence that moment-to-moment variation in positive emotions buffer genetic risk for depression: a momentary assessment twin study. Acta Psychiatr Scand 2007:115:451-7.

48. Höhn P, Menne-Lothmann C, Peeters F, et al. Moment-to-moment transfer of positive emotions in daily life predicts future course of depression in both general population and patient samples. PLoS One 2013;8:e75655

49. Glasgow N, Pillemer K, Moen P, et al; Social integration in the second half of life. Baltimore: Johns Hopkins University Press, 2000.

50. Lett HS, Blumenthal JA, Babyak MA, et al. Social support and coronary heart disease: epidemiologic evidence and implications for treatment. Psychosom Med 2005;67:869-78.

51. Constantino R, Kim Y, Crane PA. Effects of a social support intervention on health outcomes in residents of a domestic violence shelter: a pilot study. Issues Ment Health Nurs 2005;26:575-90.

52. Cohen S, Janicki-Deverts D. Can We improve our Physical Health by Altering Our Social Networks? Perspect Psychol Sci 2009;4:375-8.

53. Weinreb L, Savageau JA, Candib LM, et al. Screening for childhood trauma in adult primary care patients: a cross-sectional survey. Prim Care Companion J Clin Psychiatry 2010;12:PCC.10m00950..

54. Witt PL, McGrain P. Comparing two sample means t tests. Phys Ther 1985;65:1730-3.

55. McHugh ML. The chi-square test of independence. Biochem Med 2013;23:143-9. 